\title{
Biophysical and preliminary crystallographic studies of Lectin from Entada rheedii seeds
}

\author{
Sanjay Naik, Sanjit Kumar \\ Centre For Bioseparation Technology, VIT Vellore 632014, Tamil Nadu. India. \\ sanjitkrroy@gmail.com
}

A novel lectin was isolated, purified and characterized from seeds of Entada rheedii using ammonium sulphate precipitation followed by lactose affinity chromatography. On SDS-PAGE, the purified Entadin lectin appeared as a single band (monomer in nature) with a molecular mass of approx. $20 \mathrm{kDa}$ both in reducing as well as in nonreducing conditions. Mass spectroscopic analysis confirms the molecular weight of Entadin lectin as $19333 \mathrm{Da}$.

Entadin lectin showed highest titer value in agglutination against human blood group-B RBC and its Hemagglutination activity was inhibited by lactose, cellobiose, and galactose only. Periodic Acid Schiff's (PAS) stain confirmed the glycoprotein nature of Entadin lectin with an approx. $5 \%$ of carbohydrate content. The lectin is highly stable even after incubation at a wide range of temperatures (30 to $60{ }^{\circ} \mathrm{C}$ ) and $\mathrm{pH}$ (6 to 10). Antiproliferative effect of Entadin lectin against lung cancer cells A549 and cervical cancer cells HeLa showed $\mathrm{IC}_{50}$ value of $38 \mu \mathrm{g} / \mathrm{mL}$ and $34 \mu \mathrm{g} / \mathrm{mL}$ and no anti-proliferative activity against normal cells. Cell morphological studies revealed that Entadin lectin induced apoptosis both in A549 and HeLa cancer cells which was confirmed by (AO/EB) and Hoechst (33258) nuclear counter staining. Further, Lectin was crystallized using the hangingdrop vapour-diffusion method with $30 \%$ PEG 8000 as precipitating agent, $0.2 \mathrm{M}$ ammonium sulphate and $0.1 \mathrm{M}$ sodium cacodylate $\mathrm{pH} 6.5$.

Keywords: Lectin, Lactose affinity chromatography, Glycoprotein, Antiproliferative, crystallization 